J. Austral. Math. Soc. Ser. B 30(1989), 378-388

\title{
AN ANALYSIS OF THE IMPACT OF T. M. CHERRY'S WORK ON ASYMPTOTIC EXPANSIONS
}

\author{
R. GRIMSHAW ${ }^{1}$
}

(Received 15 August 1988; revised 7 September 1988)

\begin{abstract}
During the late 1940s, T. M. Cherry published a series of research papers on uniform asymptotic formulae for transition points in ordinary differential equations. This work, together with his research into transonic gas flows, for which it was a necessary precursor, is probably his best known and most widely quoted piece of research. An analysis is made of the impact of Cherry's work on subsequent developments in this field, both in comparison to the work of others and with respect to Cherry's work on other topics.
\end{abstract}

\section{Introduction}

T. M. Cherry was one of Australia's most distinguished mathematicians. He was professor of mathematics at the University of Melbourne from 1929 until his retirement at the end of 1963 . Throughout this period his contributions to Australian mathematics were immense, and have been documented by E. R. Love [11] in his obituary of Cherry in the Journal of the Australian Mathematical Society in 1969; see also the Royal Society obituary by K. E. Bullen and G. Temple [1]. Suffice it to mention here that Cherry was an early president of the Australian Academy of Science, and the foundation president of the Australian Mathematical Society. A list of Cherry's publications, reproduced from [11], is included at the end of this article and is here referenced as [C1], [C2], etc. Apart from the first eleven of these papers, which originated during Cherry's period at the University of Cambridge, this list describes Cherry's research while based in Australia. It is apparent that Cherry had wide interests, although I suspect that his favourite topics were dynamics and Hamiltonian systems, the research areas

\footnotetext{
${ }^{1}$ School of Mathematics, University of N.S.W., P.O. Box 1 Kensington, N.S.W. 2033 Australia

(C) Copyright Australian Mathematical Society 1989, Serial-fee code 0334-2700/89
} 
in which he commenced his career and to which he returned on a number of occasions. Indeed much of his work in this area has relevance today with the current surge of interest in nonlinear dynamical systems.

Cherry is undoubtedly best known for his work on asymptotic expansions ([C19]-[C23]) and transonic flow ([C24]-[C33]) which represent a very productive period spanning the late 1940's and 1950's. His work on uniform asymptotic formula for differential equations with turning points is undoubtedly his most widely quoted piece of work, although it would seem that Cherry himself regarded this research as simply a necessary precursor to his work on transonic flow, and having obtained the formula he needed, he did not take a serious interest in this field again. This is in spite of the fact that Cherry's papers on asymptotic expansions, together with the paper by R. E. Langer [10] published simultaneously and independently, sparked interest in this topic which continued unabated for over 20 years, and to a lesser extent, it is still an active research area today.

The purpose of this short article is to examine the impact of Cherry's work on subsequent developments in this field. Even today, Australia's geographical isolation colours our perception of the world-wide impact of Australian-based research. The preliminary (and, as will be seen, rather cursory) analysis described in this article is an attempt to shed some light on this issue from an historical perspective. Unfortunately more questions are raised than can be answered and it is to be hoped that eventually more penetrating and in-depth studies of Australian mathematicians will be made than I can possibly present here.

The methodology for the analysis of Cherry's impact consists of two parts. First the Science Citation Index (1955-1986) was searched to compile a list of all those authors who cited one of Cherry's papers [C19]-[C23] on asymptotic expansions. Then each paper in which Cherry had been cited was examined to determine the context of the citation, with the aim of distinguishing between citations which were simply acknowledgements of Cherry's early work in this field, and citations which correspond to a serious attempt to use Cherry's ideas. Since the Science Citation Index was not available before 1955, we made some effort to fill the gap 1950-1954 by checking likely journals and authors. Although this procedure can, at best, be regarded as a preliminary effort, it was sufficiently revealing to motivate the writing of this short article.

Turning first to the papers on asymptotic expansions ([C19]-[C23]), we located 63 citations. By far the largest number of citations (51) was for paper [C20], and consequently in Section 2, I shall spend some time summarising its contents. By contrast there were 21 citations for the papers on the flow of gases ([C24]-[C33]) and 5 citations for the papers on Hamiltonian systems ([C7]-[C11]) (although here, of course, the reader should be reminded that these papers were published in the 1920's and we did not search for citations prior to 1955). It 
seems clear that, at least in terms of this criterion, Cherry's paper [C20] on uniform asymptotic formulae for turning point problems, was the one which has had the most impact, although it is possible that a more objective in-depth analysis might come to a different conclusion. In 1949, almost simultaneously with the publication of Cherry's paper, R. E. Langer published a paper [10] on the same subject but using a different technique, which has received 75 citations. As far as I can ascertain, neither author was aware of the other's work at the time of publication. Certainly, neither author references the other. Previously, in 1931 and 1932, Langer had published two papers ([8] and [9]) on the same topic, which together received a further 84 citations. Of course, Cherry was aware of this, and other earlier work. Rather surprisingly, of the total of 159 citations for the three papers by Langers, only 23 were common with the Cherry citations. This suggests that there was a divergence of schools of thought in the subsequent development of the topic, and although I have not explored this issue in any detail, there does seem to be some evidence to suggest that this may be so, at least to a small degree.

\section{Summary of the paper on asymptotic expansions for turning point problems}

Here I propose to summarise Cherry's approach to this problem, as described in his paper [C20]. In the main I shall follow Cherry's development and notation although there will be some small differences to make the presentation as concise as possible. Consider the second-order ordinary differential equation

$$
d^{2} y / d x^{2}+\left(-\lambda^{2} p(x)+r\left(x, \lambda^{-2}\right)\right) y=0,
$$

for $|\lambda| \rightarrow \infty$ (in some sector of the complex $\lambda$-plane), where $p, r$ are analytic functions of $x$, and, for sufficiently large $|\lambda|, r=\sum_{0}^{\infty} r_{n}(x) \lambda^{-2 n}$. Note that first-derivative terms can be removed by standard transformations.

The classical case occurs when $p(x) \neq 0$, and then we obtain the well-known WKBJ formula

$$
y \sim p^{-\frac{1}{4}} \exp \left( \pm \lambda \int^{x} p^{\frac{1}{2}} d x\right), \quad \text { as }|\lambda| \rightarrow \infty
$$

Cherry's concern was to obtain uniform expansions as $|\lambda| \rightarrow \infty$, valid in domains which include points where $p=0$. Cherry called these points "transition points", although the terminology "turning points" is now in universal use. Cherry's procedure was to reduce the equation to a manageable form by successive transformations. Suppose that $x=0$ is a turning point, where $p(0)=0$, but $d p / d x \neq 0$ at $x=0$. Let

$$
x=\phi(z), \quad y=w\left(\phi^{\prime}\right)^{\frac{1}{2}},
$$


where $\phi^{\prime}$ is $d \phi / d z$. Then

$$
d^{2} w / d z^{2}+\left(-\lambda^{2} p \phi^{2}+r \phi^{2}+\{\phi, z\}\right) w=0
$$

where

$$
\{\phi, z\}=\phi^{\prime \prime \prime} /\left(2 \phi^{\prime}\right)-3 \phi^{\prime \prime 2} /\left(4 \phi^{\prime 2}\right) \text {. }
$$

Now choose $p \phi^{\prime 2}=z$, and it can then readily be shown that $\phi$ is an analytic function of $z$ in some domain including $z=0$. Hence we can assume that the equation to be considered has the form

$$
d^{2} w / d z^{2}+\left(-\lambda^{2} z+g\left(z, \lambda^{-2}\right)\right) w=0
$$

where $g$ is an analytic function of $z$, and $g=\sum_{0}^{\infty} g_{n}(z) \lambda^{-2 n}$ for sufficiently large $|\lambda|$.

Langer [9], [10] had shown that

$$
w \sim C A i\left(\lambda^{2 / 3} z\right)+D A i\left(\lambda^{2 / 3} e^{ \pm 2 \pi i / 3} z\right), \quad \text { as }|\lambda| \rightarrow \infty,
$$

where $\operatorname{Ai}(z)$ is the Airy function of the first kind. Cherry's aims were to improve this result in three ways. Firstly, he improved the asymptotic approximation to the stage where the error is relatively $O\left(\lambda^{-2 n-1}\right)$ for arbitrarily large integers $n$. Secondly, he established results valid uniformly for $z$ in as large a domain as possible, including unbounded domains. Thirdly, he obtained accurate formulae for $J_{\nu}(v x), x \approx 1$, where $J_{\nu}$ is a Bessel function, and for certain hypergeometric functions occurring in compressible fluid flow. This last aim was clearly the main motivation for Cherry's interest in the turning point problem, and perhaps explains why he did not pursue it again in the generality of this particular paper. Cherry also considered an alternative formulation to (2.6) in which the leadingorder approximation (2.7) is replaced by an expression involving Bessel functions of order $1 / 3$.

For (2.6), Cherry supposed that $g=\sum_{0}^{\infty} g_{n}(z) \lambda^{-2 n}$ as $|\lambda| \rightarrow \infty$, where $g_{n}(z)$ is analytic in a domain $D$, which could extend to infinity in certain sectors. Further, as $|z| \rightarrow \infty, g \sim \alpha z$. With these hypotheses, Cherry introduced a transformation,

$$
\begin{gathered}
\varsigma=\psi\left(z, \lambda^{-2}\right)=z+\sum_{1}^{\infty} \phi_{n}(z) \lambda^{-2 n} \\
\eta=w\left(\psi^{\prime}\right)^{1 / 2}
\end{gathered}
$$

Then

$$
d^{2} \eta / d \varsigma^{2}+\left(-\lambda^{2} \varsigma+G\right) \eta=0
$$

where

$$
\psi^{\prime 2} G=\lambda^{2}\left(\psi \psi^{\prime 2}-z\right)+g-\{\psi, z\}
$$


and we recall that $\{\psi, z\}$ is defined by (2.5). Now choose $\psi$ so that $G$ is as small as possible. In fact, Cherry shows that $\phi_{n}(z)$ can be chosen so that $\psi\left(z, \lambda^{-2}\right)$ is analytic in $D$, and

$$
|G| \leq A_{n}|\lambda|^{-2 n}\left\{1+|\zeta|^{7 / 2}\right\}^{-1} \text {. }
$$

The novel feature of Cherry's analysis is the construction of the near-identity transformation (2.8).

The rest of the analysis proceeds along what would now be recognised as standard lines. Thus let

$$
\eta_{1}=A i\left(\lambda^{2 / 3} \varsigma\right), \quad \eta_{2}=A i\left(\lambda^{2 / 3} \varsigma e^{ \pm 2 \pi i / 3}\right),
$$

which, of course, are the leading-order approximations of (2.10). Then from (2.10) it follows that

$$
\eta=\eta_{j}+\frac{1}{\Delta} \int_{\varsigma 0}^{\varsigma}\left\{\eta_{2}(\varsigma) \eta_{1}(t)-\eta_{1}(\varsigma) \eta_{2}(t)\right\} G(t) \eta(t) d t
$$

where $\Delta$ is the Wronskian of $\eta_{1,2}\left(\eta_{1}^{\prime} \eta_{2}-\eta_{2}^{\prime} \eta_{1}\right)$ and is a constant. Now, with a suitable choice of $s_{0}$ and the contour of integration, Picard iteration yields the desired result,

$$
\eta=\eta_{j}\left\{1+O\left(\lambda^{-2 n-1}\right)\right\}, \quad j=1,2 .
$$

This remarkably simple result is valid throughout the domain $D$, with the exclusion of certain "shadow zones". For instance with $j=1(2)$ the shadow zone is defined so that $u=\frac{2}{3} \varsigma^{3 / 2}$ cannot be joined to $u=\infty\left(\infty e^{ \pm \pi i}\right)$ by a path in $D$ whose slope (towards $\infty$ ) is everywhere between $\pi / 2(-\pi / 2)$. Cherry was apparently the first to notice the necessity for these shadow zones. Throughout, the proof is marked by the attention to detail, intricate analysis and determination to obtain the best possible result, typical of Cherry's work. Indeed, for the second-order differential equation (2.6), the scope of Cherry's result can hardly be improved, although, of course, there have been a number of extensions for the more general equation (2.1). Cherry's technique encounters minor difficulties near the zeros of $\eta_{1,2}$, but he shows how these may be overcome provided $z$ remains bounded.

For comparison, I shall now describe briefly the results obtained by Langer [10], for simplicity restricting attention to the case when the equation to be considered is again (2.6). In Langer's method, we introduce two approximate solutions for $w$,

$$
w_{j}=A\left(z, \lambda^{-2}\right) \eta_{j}+\left(B\left(z, \lambda^{-2}\right) / \lambda^{2}\right) \eta_{j}^{\prime}, \quad j=1,2 .
$$

Substitution into (2.16) then gives

$$
\left.\begin{array}{c}
d^{2} w_{j} / d z^{2}+\left(-\lambda^{2} z+g\right) w_{j}=S_{0} \eta_{j}+S_{1} \eta_{j}^{\prime} \\
S_{0}=g A+A^{\prime \prime}+B+2 z B^{\prime} \\
S_{1}=2 A^{\prime}+\lambda^{-2}\left(B^{\prime \prime}+g B\right) .
\end{array}\right\}
$$


Now let $A=1+\sum_{0}^{m} a_{n}(z) \lambda^{-2 n}, B=\sum_{0}^{m} b_{n}(z) \lambda^{-2 m}$, and choose the coefficients so that $S_{0}, S_{1}$ are $O\left(\lambda^{-2 m-2}\right)$. The remainder of the construction is similar to Cherry's, in that an integral equation analogous to (2.14) is formed, and solved by Picard iteration. However, Langer restricted $z$ to be real and bounded, although later Olver [13] showed that Langer's method can be extended to allow $z$ to be complex and to lie in unbounded domains. The Cherry and Langer methods give equivalent asymptotic expansions near the turning point $z=0$. It should also be noted that Langer considered the more general equation

$$
d^{2} w / d z^{2}+\left(-\lambda^{2} z+\lambda h(z)+g\left(z, \lambda^{-2}\right) w=0\right.
$$

which he analysed in a similar way to (2.6), although the technical details are much more complicated.

\section{Discussion}

Following the simultaneous publication of the Cherry and Langer papers, there was an explosive growth of interest in the turning point problem. This is not the place to give a comprehensive survey of the history of the turning point problem, although a complete listing of the Cherry and Langer citations would form a useful base for such a survey. My purpose here is to report the results of an analysis of the Cherry citations, with the main aim of distinguishing between citations which were simply an acknowledgement of Cherry's early work, and citations which were related to a direct use of Cherry's results and techniques. Of the 51 citations of the paper [C20], 27 were in the latter category, and these were spread evenly over the period 1950-1986.

In looking at the Cherry citations in more detail, I should again caution the reader that $I$ am not attempting to give a balanced assessment of the value of subsequent work. Instead, my intention is merely to comment on the relation between subsequent research and the pioneering work of Cherry and Langer, with the emphasis here naturally on Cherry. In a series of papers which began in the 1950's Olver, at that time in the U.K., gave an exhaustive and detailed account of the turning point problem and various extensions (see [13] for two of the early significant papers in this sequence, and [14] for Olver's textbook which summarises much of his work). Olver used the Langer method for (2.6), and in particular extended it to allow $z$ to lie in a complex domain, which could be unbounded, although he required $g(z) \sim \alpha z^{-2}$ as $|z| \rightarrow \infty$, whereas Cherry allowed $g \sim \alpha z$. Olver was aware of Cherry's work but does not seem to have made any significant use of his results or technique. Like Cherry he obtained results for $J_{\nu}(\nu x), x \approx 1$. He also extended the theory to the case when a turning point was close to a singularity (i.e. $p(x)$ in (2.1) has a zero in a domain in which 
either $p$ or $r$ also has a pole-type singularity), and was able to obtain explicit error bounds for his asymptotic expansions. Also, Thorne [16], an Australian research student at Cambridge, considered the case when there was a turning point close to a singularity, and applied his results to Legendre functions. He used a method similar to Langer and Olver, but was aware of Cherry's work, and in particular, Cherry's related results for hypergeometric functions.

In the U.S.A., Langer continued to work in the area and published a number of papers throughout the 1950's, in particular, looking at higher-order equations. Also in the U.S.A., Erdelyi refined and extended results for the turning point problem and published what I believe to be one of the first textbooks [6] in the area. Erdelyi was well aware of Cherry's work, and particularly in his early papers, made some use of the Cherry technique. However, undoubtedly the most prolific output in the U.S.A. in the 1950's and 1960's came from the N.Y.U. school, and is associated with such names as Wasow [17] and J. B. Keller. This work generally used the Langer method for extensions to higher-order equations, systems of differential equations, several turning points, turning points close to singularities, higher-order turning points and so on. A useful review of this work was given by McHugh [12], which, however, has only a brief mention of Cherry's work. Although the N.Y.U. school largely used the Langer method, an interesting exception was Zauderer [18], [19] who made a spirited defence of the Cherry technique, pointing out its advantages over the Langer method, and showing how it could also be extended to higher-order turning points and so on.

Turning to more recent times, most of the Cherry citations relate to the utilisation of the turning-point theory for the solution of eigenvalue problems for the Schrödinger equation, with applications to theoretical chemistry. These papers are evenly divided between work originating in the U.S.A., and U.S.S.R. Both groups make extensive use of the Cherry method which appears to be particularly advantageous for eigenvalue problems. The Russian group appears to have originated with the work of Slavyanov in the 1950's and 1960's, and interestingly sometimes refer to the collected works of T. M. Cherri [sic], translated into Russian.

Next we turn to an analysis of some of the textbooks on asymptotic expansions ([2]-[7], [14], [15], [17]). Science Citation Index does not record book citations and this list is simply those known to me. References [5] and [7] make no mention of either Cherry or Langer, and [15] mentions only the Langer method. The remaining texts all use the Langer method for their account of the turning point problem, although Cherry's work is acknowledged as being useful for unbounded domains. Only the text by Erdelyi gives a description of the Cherry technique.

To summarise, it would seem that Cherry's work was known to a substantial number of the subsequent workers in the field, including all the major figures, and his technique is currently being exploited by at least two groups working on 
eigenvalue problems which arise in theoretical chemistry. However, in general it would seem that the Langer method has been preferred, particularly since Olver [13] has shown that it can be extended to complex $z$ in unbounded domains. The essential difference between the two methods can be loosely stated as that, whereas Cherry expands in the phase, Langer expands in the amplitude. To date the Langer method appears to be more easily generalised, although as we noted above, the Cherry method has been found useful for eigenvalue problems. There are a number of unanswered questions which arise out of this account of Cherry's work on turning points, most notably, why Cherry did not return to this topic after his initial work on it, and what contact, if any, did Cherry have with subsequent workers in this field. An examination of the Cherry archives in the University of Melbourne throws little direct light on this, although it is clear that Cherry's major interest at the time was fluid dynamics, and particularly transonic flow. Those who worked with Cherry at the time say that he had a reputation for working alone, rather than by interaction. Nevertheless, it is to be hoped that ultimately some of these aspects will be addressed more deeply by mathematical historians.

\title{
Acknowledgement
}

It is a pleasure to record the assistance of Fariday Mahfoz in the analysis of the Science Citation Index. I also wish to thank Professor E. R. Love for permission to reproduce his bibliography of Cherry.

\author{
Publications by T. M. Cherry \\ (reproduced from [11])
}

\section{Ordinary Differential Equations}

[C1] "On the solution of difference equations", Proc. Cambridge Phil. Soc. 21 (1923) 711729.

[C2] "On the form of the solution of the equations of dynamics", Trans. Cambridge Phil. Soc. 23 (1924) 43-70

[C3] "Integrals of systems of ordinary differential equations", Proc. Cambridge Phil. Soc. 22 (1924) 273-281.

[C4] "On Poincare's theorem on the non-existence of uniform integrals", Proc Cambridge Phil. Soc. 22 (1924) 287-294.

[C5] "Note on the employment of angular variables in celestial mechanics", M. N. RoyAstronomical Soc. 84 (1924) 729-731. 
[C6] "Some examples of trajectories defined by differential equations of a generalized dynamical type", Trans. Cambridge Phil. Soc. 23 (1925) 169-200.

\section{Hamiltonian Systems of Differential Equations}

[C7] "On integrals developable about a singular point of a Hamiltonian system of differential equations", Proc. Cambridge Phil. Soc. 22 (1924) 325-349.

[C8] "On integrals developable about a singular point of a Hamiltonian system of differential equations, part II", Proc. Cambridge Phil. Soc. 22 (1925) 510-533.

[C9] "On the transformation of Hamiltonian systems of linear differential equations with constant or periodic coefficients", Proc. London Math. Soc. [2] 26 (1926) 211-230.

[C10] "On the solution of Hamiltonian systems of differential equations in the neighbourhood of a singular point", Proc. London Math. Soc. [2] 27 (1927) 151-170.

[C11] "On periodic solutions of Hamiltonian systems of differential equations", Phil. Trans. Roy. Soc. London A 227 (1928) 137-221.

\section{Topological Dynamics}

[C12] "Topological properties of the solutions of ordinary differential equations", American Journal of Math. 59 (1937) 957-982.

[C13] "Analytical quasi-periodic curves of discontinuous type on a torus", Proc. London Math. Soc. [2] 44 (1938) 175-215.

\section{Later Dynamical Studies}

[C14] "The pathology of differential equations", J. Australian Math. Soc. 1 (1959), 1-16.

[C15] "A singular case of iteration of analytic functions: a contribution to the small divisor problem", in Non-linear Problems of Engineering. (Academic Press, 1964), 29-50.

[C16] "Asymptotic solutions of analytic Hamiltonian systems", J. Differential Equations 4 (1968) 142-159.

\section{Fourier-type Expansion Theorems}

[C17] "On expansions in eigenfunctions, particularly in Bessel functions", Proc. London Math. Soc. [2] 51 (1948) 14-45.

[C18] "Expansions in terms of parabolic cylinder functions", Edinburgh Math. Soc. Proc. [2] 8 (1948) 50-65.

Asymptotic Expansions

[C19] "Uniform asymptotic expansions", J. London Math. Soc. 24 (1949) 121-130.

[C20] "Uniform asymptotic formulae for functions with transition points", Trans. American Math. Soc. 68 (1950) 224-257. 
[C21] "Asymptotic expansions for the hypergeometric functions occurring in gas flow theory", Proc. Roy. Soc. A 202 (1950) 507-522.

[C22] "Tables and approximate formulae for hypergeometric functions, of high order, occurring in gas flow theory", Proc. Roy Soc. A 217 (1953) 222-234.

[C23] Summation of slowly convergent series", Proc. Cambridge Phil. Soc. 46 (1950) 436-449.

\section{Flow of Gases}

[C24] "Flow of a compressible fluid about a cylinder", Proc. Roy. Soc. A 192 (1947) 45-79.

[C25] "Flow of a compressible fluid about a cylinder, Part II: Flow with circulation", Proc. Roy. Soc. A 196 (1949) 1-31.

[C26] "Numerical solutions for transonic flow", Proc. Roy. Soc. A 196 (1949) 32-36.

[C27] "Numerical solutions for compressible flow past a cylinder", C.S.I.R. (Australia) Div. of Aeronautics Rep. A 48 (1949) 1-25.

[C28] "Exact solutions for flow of a perfect gas in a two-dimensional Laval nozzle", Proc. Roy. Soc. A 203 (1950) 551-571.

[C29] "Relation between Bergman's and Chaplygin's methods of solving the hodograph equation", Q. Applied Math. 9 (1951) 92-94.

[C30] "A transformation of the hodograph equation and the determination of certain fluid motions", Phil. Trans. Roy. Soc. London A 245 (1953) 583-624.

[C31] "Some nozzle flows found by the hodograph method", J. Australian Math. Soc. 1 (1959) $80-94$.

[C32] "Some nozzle flows found by the hodograph method, part II", J. Australian Math. Soc. 1 (1960) 357-367.

[C33] "Trans-sonic nozzle flows found by the hodograph method", in Partial Differential Equations and Continuum Mechanics, (Univ. of Wisconsin Press, 1961) 217-232.

\section{Miscellaneous Topics}

[C34] "The value of inoculation-a statistical inquiry", in Influenza and Maritime Quarantine in Australia by J. H. L. Cumpston (C'wealth of Australia, 1919) 89-103.

[C35] "Newton's Principia in 1687 and 1937: a lecture", Melbourne University Press (1937) 7-28.

[C36] "General theory of the magnetron", C.S.I.R. (Australia) Radiophysics Lab., Rep. MUM 1 (1943) 1-24.

[C37] "Probability of detection of aircraft by radio direction finding", C.S.I.R. (Australia) Radiophysics Lab., Rep. MUM 2 (1943) 1-28.

[C38] "Probability of detecting a radar station from an aircraft", C.S.I.R. (Australia) Radiophysics Lab., Rep. MUM 3 (1944) 1-16.

[C39] "Flow and generation of heat in compressed films of viscous liquid", C.S.I.R. (Australza) Div. of Lubricants and Bearings, Explosives Rep. No. 8 (1945) 79 pages.

[C40] "A lesson on number", Australian Mathematics Teacher 9 (1947) 33-41, 65-74.

[C41] "On Kepler's equation", Proc. Cambridge Phil. Soc. 51 (1955) 81-91.

[C42] (With P. E. Lush) "The variational method in hydrodynamics", Q. J. Mech. Appl. Math. 9 (1956) 6-21.

[C43] "Numerical solution of a problem in forced convection", Proc. W. R. E. Computing Conference. (Weapons Research Establishment, Adelaide, 1957) 115-1 to 115-13.

[C44] "A mathematician looks at physical theory: presidential address to ANZAAS Section A", Australian J. Science 21 (1958) 17-27. 
[C45] "Steady motions related to problems of hydrodynamic stability", Australian Math. Soc. Summer Res. Inst., Rep. II (1961) IV-1 to IV-8.

[C46] (With B. H. Neumann) "Felix Adalbert Behrend", J. Australian Math. Soc. 4 (1964) 264-270.

[C47] "Infinite linear systems with homogeneous kernel of degree -1", J. Australian Math. Soc. 5 (1965) 129-168.

\section{References}

[1] K. E. Bullen and G. Temple, "Thomas MacFarland Cherry, 1898-1966", Biog. Mem. Fellows Roy. Soc. 14. (1968) 117.

[2] L. Cesari, Asymptotic behavior and stablity in ordinary differential equations (Springer, Berlin, 1962).

[3] E. Copson, Asymptotic expansions (CUP, Cambridge, 1965).

[4] R. Dingle, Asymptotic expansions: therr derivation and interpretation (Academic Press, New York, 1973).

[5] N. de Bruijn, Asymptotic methods in analysis (North-Holland, Amsterdam, 1958).

[6] A. Erdèlyi, Asymptotic Expansions (Dover, New York, 1956).

[7] H. Jeffreys, Asymptotic Approximations (Dover, New York, 1962).

[8] R. E. Langer, Trans. Amer. Math. Soc., 33 (1931) 23.

[9] R. E. Langer, Trans. Amer. Math. Soc., 34 (1932) 447.

[10] R. E. Langer, Trans. Amer. Math. Soc., 67 (1949) 461.

[11] E. R. Love, "Thomas MacFarland Cherry", J. Aust. Math. Soc., 9 (1969) 1.

[12] J. A. M. McHugh, Arch. Hist. Ex. Sc., 7 (1971) 277.

[13] F. W. J. Olver, Phil. Trans. Roy. Soc., A247 (1954) 307,328.

[14] F. W. J. Olver, Asymptotics and special functions (Academic, New York, 1974).

[15] L. Sirovich, Techniques of asymptotic analysis (Springer, Berlin, 1971).

[16] R. C. Thorne, Phil. Trans. Roy. Soc. A249 (1957) 585, 597.

[17] W. Wasow, Asymptotic expansions for ordinary differential equations (Interscience, New York, 1965).

[18] E. Zauderer, Proc. Amer. Math. Soc., 31 (1972) 489.

[19] E. Zauderer, J. Chem. Phys., L56 (1972) 5198. 